\title{
ERRATUM
}

Ibrahim A. Abbas

\section{Generalized magneto-thermoelasticity in a nonhomogeneous isotropic hollow cylinder using the finite element method}

Published online: 3 February 2009

(C) Springer-Verlag 2009

Erratum to: Arch Appl Mech (2009) 79: 41-50

DOI 10.1007/s00419-008-0206-9

With regret, the original article was published with errors.

1. In line 94 page 3 , the equation number (9) ( $T$ change to $\frac{\partial T}{\partial r}$ ) to become

$$
\frac{1}{r} \frac{\partial}{\partial r}\left(r K \frac{\partial T}{\partial r}\right)=\rho C_{v}\left(\frac{\partial}{\partial t}+t_{0} \frac{\partial^{2}}{\partial t^{2}}\right) T+\gamma T_{o}\left(\frac{\partial}{\partial t}+n t_{0} \frac{\partial^{2}}{\partial t^{2}}\right)\left(\frac{\partial u}{\partial r}+\frac{u}{r}\right) .
$$

2. In line 111 and 112 page 4 , the equation number (16) ( $\varepsilon$ change to $\left.\varepsilon_{c}\right)$ to get

$$
\frac{\partial^{2} T}{\partial r^{2}}+\frac{2 m+1}{r} \frac{\partial T}{\partial r}=\left(\frac{\partial}{\partial t}+t_{0} \frac{\partial^{2}}{\partial t^{2}}\right) T+\varepsilon_{c}\left(\frac{\partial}{\partial t}+n t_{0} \frac{\partial^{2}}{\partial t^{2}}\right)\left(\frac{\partial u}{\partial r}+\frac{u}{r}\right),
$$

where $\xi^{2}=\frac{\lambda_{*}+2 \mu_{*}}{\mu_{*}}, \omega^{2}=\frac{\mu_{o *} H_{o}^{2}}{\mu_{*}}, \eta=\frac{T_{o} \gamma_{*}}{\mu_{*}}, \varepsilon=2\left(m-\frac{\mu_{*}}{H_{a}}\right)-1, \beta=\frac{T_{o} \gamma_{*}}{H_{a}}, \varepsilon_{c}=\frac{\gamma_{*}}{\rho_{*} C_{v}}$. 